\title{
AVALIAÇÃO DA PRÁTICA DE ATIVIDADE AERÓBIA E NÍVEL DE CONHECIMENTO DOS SEUS PRATICANTES
}

\author{
Evaluation of the practice of aerobic activity and level \\ of knowledge of its participants
}

\section{Fernanda Gonçalves Gomes Alvarez ${ }^{1}$, José Carlos Ferreira Reis ${ }^{2,3}$, Maurício Garcia Ennes ${ }^{2,3}$}

\section{Resumo}

Baseado em um questionário aplicado, foi realizado um estudo em que foram analisados o tempo e a freqüência semanal dedicados à prática da atividade aeróbia, por indivíduos do sexo feminino, mensurando-se, também, o nível de conhecimento das praticantes com relação à atividade. Por meio deste estudo, foi possível constatar que, apesar de $59 \%$ das entrevistadas se sentirem dentro dos limites considerados normais, no que se refere à gordura, $56 \%$ buscam, na atividade aeróbia, o emagrecimento. $60 \%$ das entrevistadas acreditam que quanto mais tempo for dedicado a essa atividade, mais rápido seu objetivo será atingido, de forma que acabam por praticar atividade física em excesso, correndo o risco de lesão músculo-esquelética e overtraining. Mais de $40 \%$ das entrevistadas podem estar treinando em excesso. O estudo analisou, ainda, o grau de influência exercida pelo profissional de Educação Física, comparando-o a outras fontes de informação. Foi constatado que o profissional de Educação Física exerce menor influência, no que se refere à orientação dessas atividades, do que outras fontes de informação. Apenas $6,4 \%$ das entrevistadas obtiveram informação sobre a atividade aeróbia praticada através de professor de Educação Física.

Palavras-chave: Emagrecimento, Atividade Aeróbia, Nível de Conhecimento.

\begin{abstract}
Based on an applied questionnaire, a study was realized in which the weekly time and frequency dedicated to the practice of aerobic activity were analyzed, by individuals of the female sex, also measuring the level of knowledge of the practicants regarding the activity. Through this study, it was possible to establish that only $59 \%$ of those interviewed felt, themselves, to be within limits considered normal in regard to fat and $56 \%$ sought to slim through aerobic activity. $60 \%$ of those interviewed considered that the more time they dedicated to this activity, the more rapidly they would achieve their objective, in a way that would end the practice of excessive physical activity, running the risk of muscular-skeletal lesion and overtraining. More than $40 \%$ of those interviewed could be training excessively. The study also analyzed the degree of influence exercised by the Physical Education professional compared to other sources of information. It was established that the Physical Education professional exercised less influence in regard to the orientation of these activities than other sources of information. Only $6.4 \%$ of those interviewed obtained information regarding the aerobic activity practiced through the Physical Education professor.
\end{abstract}

Key words: Slimming, Aerobic Activity, Level of Knowledge.

1. Fundação Oswaldo Cruz (FIOCRUZ) - Rio de Janeiro - RJ - Brasil.

2. União das Faculdades Maria Thereza - Niterói - RJ - Brasil.

3. Conselho Regional de Educação Física - Rio de Janeiro - RJ - Brasil.

Recebido em 03.09.2007. Aceito em 16.01.2008.

Revista de Educação Física 2008;140:13-19 


\section{INTRODUÇÃO}

Atualmente, a aparência física é uma das formas mais presentes de exclusão social, principalmente para as mulheres. A imagem da mulher e do feminino continua associada à da beleza, havendo cada vez menos tolerância para os desvios dos padrões estéticos socialmente estabelecidos, sentindo-se excluídas aquelas que se encontram à margem desses limites de beleza.

Courtine (1995), citado por Novaes e Vilhena (2003), evidencia, por exemplos históricos, o fascínio e o estado de corpolatria característicos da sociedade em que vivemos. Segundo o autor, esse processo remete ao fato de que, em outros momentos históricos, a apreciação estética do corpo se dava de forma menos fragmentada, não estando em jogo, pedaços, recortes da anatomia humana, sendo valorizado um todo harmônico.

Essas modificações, que ocorreram ao longo do tempo, sempre sofreram influência da conjuntura social. Além disto, atualmente, outro fator de grande influência nas opiniões e nas atitudes das pessoas é a mídia. Assim como tudo o que é veiculado pelos meios de comunicação, o padrão de estética corporal exibido tornou-se meta dos indivíduos atingidos por suas mensagens. Desde então, aspectos que podem contribuir para aquisição e para a manutenção da estética "ideal," preconizada pela mídia, como a atividade física, têm se tornado uma rotina na vida dos indivíduos e, em especial, na vida das mulheres.

A problemática do peso, vivida especialmente pelas mulheres, parece ser estabelecida cedo na vida. $A$ participação sistemática em programas de atividade física, na vida adulta, é influenciada pelas experiências vividas durante a infância e adolescência. O período entre 25 e 44 anos representa a época mais perigosa para desenvolvimento de uma adiposidade excessiva, destacando-se as mulheres que tendem a ganhar maior quantidade de peso. Segundo McArdle, Katch e Katch (2003:845), 14\% das mulheres ganham mais de 13,62 kg, entre 25 e 34 anos.

Ao considerar que o acúmulo de gordura corporal é devido ao desequilíbrio entre o consumo e o gasto energético, torna-se imprescindível a combinação de dieta e de atividade física, binômio capaz de determinar a variação do peso corporal do indivíduo. O acréscimo do exercício, em um programa de redução, modifica, favoravelmente, a composição do peso perdido em direção a uma maior perda de gordura (McArdle et al., 2003:876).

Diversos autores, como Carpenter (2002:67), Wilmore e Costill (2001:681), McArdle, Katch e Katch (2003:878), recomendam a prática de atividades aeróbias para as pessoas que visam a diminuição de gordura corporal. Segundo Silva (1995), citado por Gagliardi (2006), a atividade física atua na composição corporal, contribuindo, decisivamente, no combate ao excesso de peso corporal. São exemplos de exercícios aeróbios: caminhada, corrida em ritmo moderado (jogging), natação, ciclismo, jogos coletivos (basquetebol, futebol, etc.) e modalidades praticadas individualmente (esportes com raquetes).

O American College of Sports Medicine (2003:141) recomenda a inclusão de atividade de endurance em programas de treinamento voltados para perda de peso. A fase ou o estímulo de endurance deve ter duração entre 20 e 60 minutos, em sessões contínuas ou intermitentes (sessões mínimas de 10 minutos, distribuídas no transcorrer do dia).

A duração depende da intensidade da atividade, ou seja, uma atividade de intensidade moderada deve ser realizada durante um período de tempo mais longo e, inversamente, indivíduos que treinam em níveis de intensidade mais altos, devem fazê-lo por períodos mais curtos (ACSM, 2003: 94).

É possível perceber que a atividade aeróbia, no âmbito das academias, vem sendo usada como principal estratégia para diminuição da massa gorda. Porém, não se sabe se os indivíduos que praticam essas atividades têm conhecimento sobre metodologias de treino que maximizem seus resultados, sem, contudo, submeter seus corpos a um estresse físico capaz de lesioná-los.

Assim, o objetivo desse estudo é verificar o nível de conhecimento e o volume de treinamento de mulheres, praticantes de atividades aeróbias, quanto ao efeito da mesma em relação ao processo de emagrecimento.

\section{METODOLOGIA}

A pesquisa foi realizada com 78 indivíduos, do sexo feminino, com idade média de 33,86 anos, peso médio de $57,92 \mathrm{~kg}$, altura média de 1,66 m e IMC médio de 20,69. Todas eram praticantes de atividade aeróbia nas seguintes academias: Academia Universo Atlético, situada em Botafogo, zona sul do Rio de Janeiro; Academia Fitness 


\section{GRÁFICO 1 \\ PERCEPÇÃO ESTÉTICA DAS ENTREVISTADAS.}

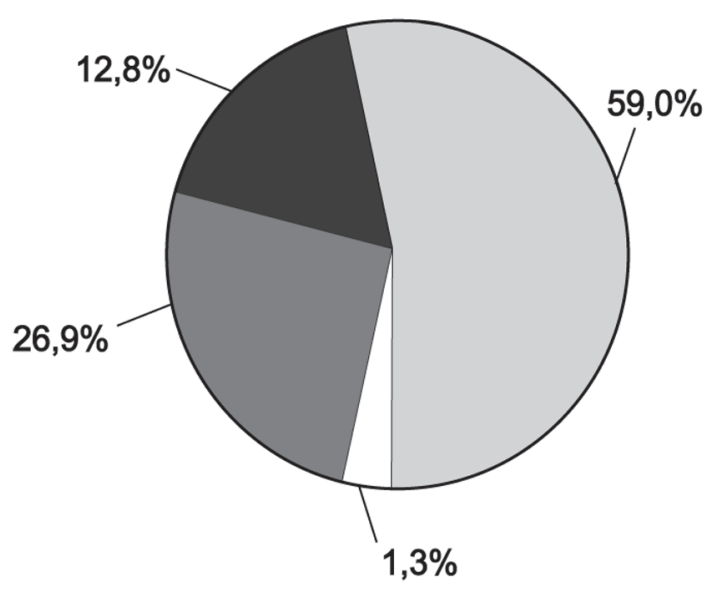

Magro

Normal

Acima do peso

Obeso

295, situada em Vila Isabel, na zona norte do Rio; e, ainda, freqüentadores da Academia da Fundação Oswaldo Cruz, localizada em Bonsucesso, no Rio de Janeiro.

A coleta de dados foi realizada através de um questionário, validado por três mestres, auto-aplicado, contendo oito questões fechadas. Foram observadas todas as normas referentes ao parecer $\mathrm{n}$ ํ196, de 10 de Outubro de 1996, do Conselho Nacional de Saúde, que versa sobre experimentos com seres humanos.

\section{RESULTADOS E DISCUSSÃO}

Quando indagadas sobre como se sentiam em relação à sua própria imagem, 59,0\% das entrevistadas responderam que se sentiam normais, dentro do peso considerado ideal; $26,9 \%$ se sentiam acima desse índice;
$12,8 \%$ se sentiam magras; e apenas $1,2 \%$ se consideravam obesas. Este resultado pode ser visto no GRÁFICO 1.

Quando indagadas sobre seus objetivos ao realizarem as atividades aeróbias, as pesquisadas mostram que há uma busca incessante de emagrecimento (GRÁFICO 2): $56,0 \%$ desejam emagrecer; $26,7 \%$ desejam apenas saúde e qualidade de vida; e os outros $17,3 \%$ desejam manter o peso atual.

Comparando os GRÁFICOS 1 e 2, nota-se que a maior parte das praticantes de atividade física sente-se normal, ou seja, dentro da faixa da normalidade quanto ao índice de gordura corporal, mas, mesmo assim, procuram emagrecer. Esse fato pode ser influenciado pela imagem que a mídia oferece a respeito de corpos, onde se investe em um jogo de espelhos entre o corpo e o olhar do outro, operando na construção da auto-estima e da auto-imagem.

GRÁFICO 2

PRINCIPAL OBJETIVO.

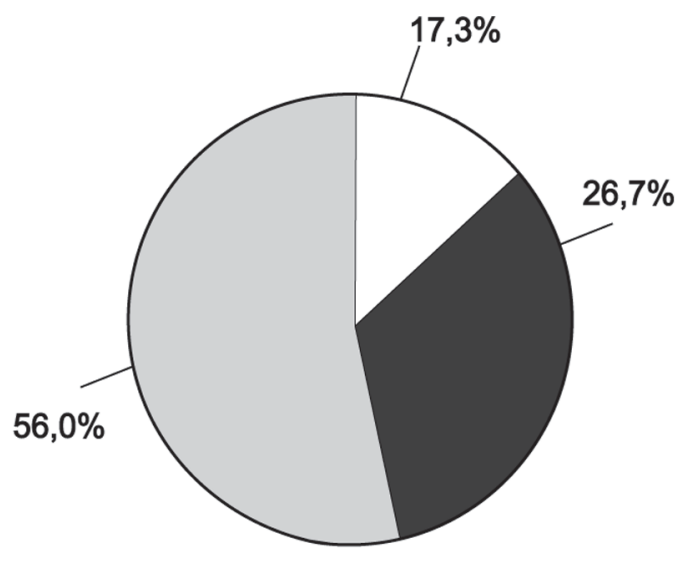

Apenas saúde e qualidade de vida

Emagrecer

Manter o peso 


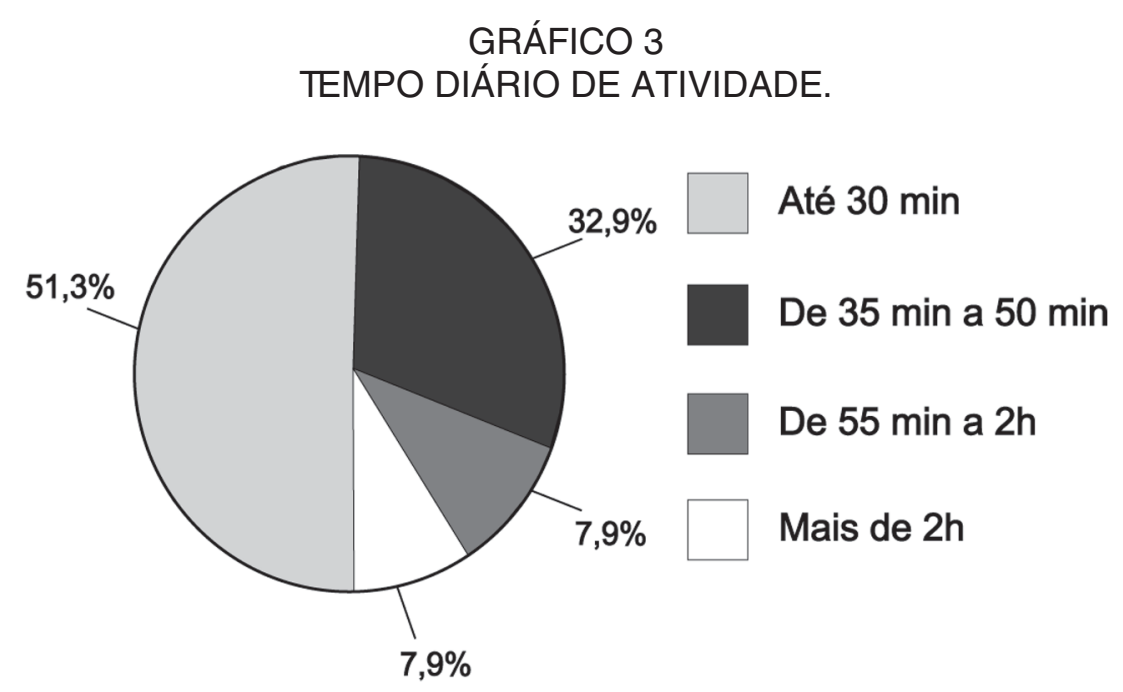

Segundo Malysse (1997), estas pessoas esforçam-se, durante todo o ano, com exercícios massacrantes, para, no verão, terem a recompensa de ir à praia expondo o corpo sem vergonha. Disciplina-se o corpo a freqüentar uma academia de ginástica, a fim de que, à custa de muito suor e calorias perdidas, consiga-se reconhecimento social e aprovação (Novaes, 2007).

Uma ótima revelação, é a busca pela saúde e pela qualidade de vida, uma consciência significativa apontada no GRÁFICO 2.

O GRÁFICO 3 pode apontar para um excesso de treino aeróbio por parte das praticantes, já que mais de $15 \%$ das entrevistadas podem estar treinando acima do recomendado: 7,9\% das entrevistadas estão treinando mais de 2 horas, tempo acima do recomendado pelo ACSM (2003:94); os outros 7,9\% poderiam estar, ou não, dentro dos parâmetros, uma vez que a faixa de 55 min a $2 \mathrm{~h}$ deixou, em sua parcialidade, de ser considerado excesso de treino pelo ASCM (2003). Torna-se, então, necessário desmembrar essa faixa em duas outras de 55 a 60min, dentro dos limites estabelecidos pelo ASCM, e outra, de $60 \mathrm{~min}$ a $2 \mathrm{~h}$, considerada excesso pelo ASCM para uma afirmação mais precisa .

Quando indagadas sobre a freqüência semanal com que praticam atividades aeróbias: $7,7 \%$ dedicam-se todos os dias; $35,6 \%$, cinco vezes na semana; $42,6 \%$, três vezes; e $14,1 \%$ das entrevistadas, duas vezes por semana (GRÁFICO 4).

Verifica-se que uma parcela significativa da amostra vem praticando esta atividade diariamente. Sabe-se que a freqüência semanal ideal, recomendada pelo ACSM (2003:92), é de três a cinco vezes por semana. Acima de

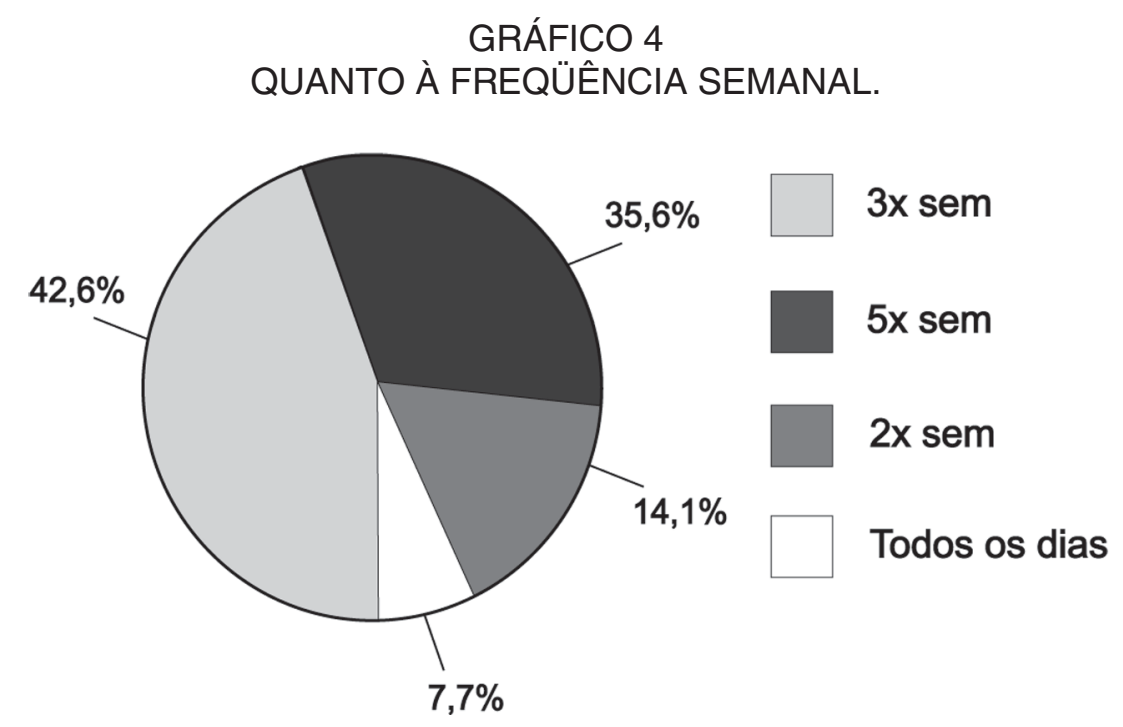


NÍVEL DE CONHECIMENTO SOBRE TREINAMENTO AERÓBIO.

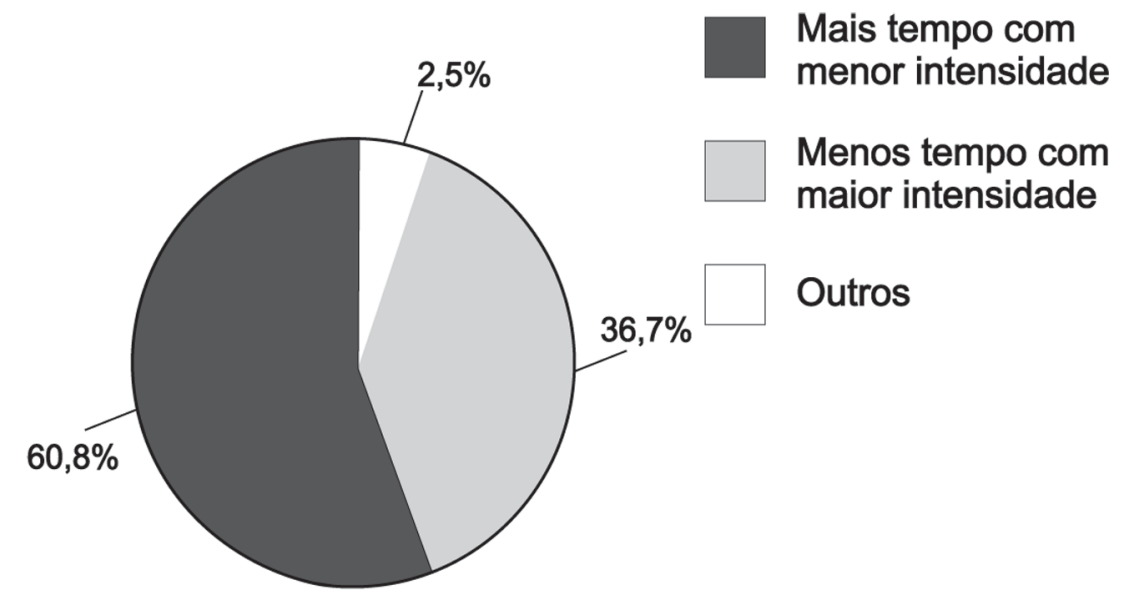

cinco vezes, o aumento de $\mathrm{VO}_{2 \max }$ é desprezível ou nulo, crescendo bastante a possibilidade de incidência de lesões músculo-esqueléticas.

De acordo com Cohen (2002), o excesso de atividade física pode desencadear a Síndrome da Fadiga Crônica, também conhecida como Síndrome do Overtraining. A Síndrome do Overtraining ocorre quando há uma diminuição do desempenho do atleta, gerada pelo estresse do treinamento, sem que haja o tempo necessário para a recuperação do organismo. O overtraining desencadeia uma série de alterações metabólicas, comprometendo o sistema cardiovascular, o neuroendócrino, o imune e o músculo-esquelético.

Quando questionadas sobre o melhor treinamento para emagrecer, tem-se, como resultado, que $60,8 \%$ acreditam que mais tempo de atividade aeróbia, em uma menor intensidade, é o melhor para emagrecer; $36,7 \%$ acreditam que o melhor é ficar menos tempo, porém em uma intensidade maior; e 2,5\% responderam outros (GRÁFICO $5)$.

Constatou-se que a maioria das praticantes acredita que realizar atividade aeróbia por mais tempo, em uma menor intensidade, é mais eficiente para o emagrecimento.

Segundo Capenter (2002:56), observando esforços de longa duração e com intensidades baixas, em contrapartida com curtas durações e altas intensidades, o custo energético foi similar em ambos os casos.

Tremblay et al. (1990), citado por Oliveira (2007), avaliaram a relação entre a intensidade da atividade física habitualmente realizada e a composição corporal de mais de 2.500 pessoas. Através de um questionário, os indivíduos foram divididos em quatro grupos, de acordo com a intensidade das atividades realizadas: menores que 5 METs, entre 5 e 7 METs, entre 7 e 9 METs e maiores que 9 METs. Apesar de não haver diferença no gasto calórico entre os grupos, os resultados demonstraram que indivíduos, habitualmente empenhados em atividades intensas, possuem menor relação cintura-quadril, assim como uma menor quantidade de gordura subcutânea.

$O$ fato de pessoas que realizam atividades intensas apresentarem menor percentual de gordura, trabalhando em intensidade fora da zona de queima de gordura, demonstra que outros fatores são determinantes para os resultados de um programa de emagrecimento, contrariando o modelo "mais tempo melhor" de emagrecimento adotado por muitos praticantes de atividades aeróbias.

Quanto à origem da informação sobre a atividade aeróbia: $19,5 \%$ das entrevistadas obtiveram a informação por meios de revistas; $20,8 \%$ ouviram falar sobre o tema; $6,5 \%$ se informaram através de um professor; e 53,2\% por outros meios distintos (GRÁFICO 6).

Esses resultados apontam para a influência maior das revistas e de outras fontes na transmissão de informação sobre a atividade aeróbia e sobre o emagrecimento, se comparada à influência do profissional de Educação Física nesse campo. Esse fato é ruim, pois pode-se perceber que o profissional de Educação Física não está cumprindo o seu papel de orientar e passar o conhecimento específico sobre o assunto. 


\section{GRÁFICO 6 \\ FORMA DE OBTENÇÃO DA INFORMAÇÃO SOBRE A ATIVIDADE AERÓBIA.}

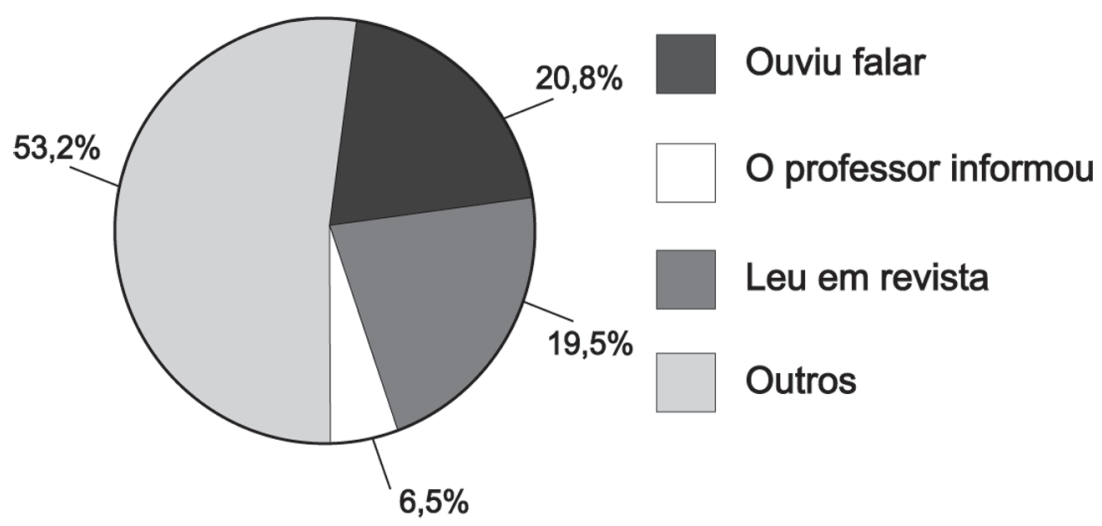

\section{CONCLUSÃO}

Em busca do emagrecimento, as entrevistadas realizam atividades aeróbias além do recomendado pelo ASCM, acreditando na premissa de que "quanto mais, melhor". Ou seja, acreditam que ao se dedicar mais tempo à atividade aeróbia, melhor atingem seu objetivo: emagrecer. Sendo assim, submetem-se a exagerados esforços, deixando seus corpos suscetíveis a lesões articulares, ósseas e musculares.

Outro dado de relevante valor, obtido pelo estudo, foi a forma de obtenção da informação sobre atividades aeróbias para o emagrecimento. O professor exerce menor influência, quando comparados com outras fontes de informação. Esse dado é preocupante, uma vez que todo aluno tem que, necessariamente, passar pelo professor, que deve prescrever, informar, explicar e, ainda, se necessário, tirar dúvidas.

Como sugestão para outros estudos, pode-se destacar a quantificação do volume de treinamento em relação a lesões músculo-esqueléticas e em relação a níveis de estresse, classificando-as quanto à proximidade do overtraining.

Seria interessante, também, a investigação sobre o porquê de outras formas de obtenção de informação exercerem maior influência sobre os alunos do que professores formados e especializados, procurando descobrir como estes últimos podem aumentar seu grau de influência.

\section{REFERÊNCIAS BIBLIOGRÁFICAS}

AMERICAN COLLEGE OF SPORTS MEDICINE. Diretrizes do ACSM para os testes de esforço e sua prescrição. Rio de Janeiro: Guanabara Koogan, 2003.

CAPETER CS. Treinamento cardiorrespiratório. Rio de Janeiro: Sprint, 2002.

COHEN M et all. Estudo das lesões musculares no esporte.São Paulo: UNIFESP, 2002.

FOX E, BOWERS RW, FOSS M. Bases fisiológicas da educação física e do desporto. 4aㅡ ed. Rio de Janeiro: Guanabara Koogan, 1991.

GAGLIARDI M. Obesidade e peso corporal: riscos e conseqüências. Disponível em: <www.unipinhal.edu.br/ movimentopercepcao/include/getdoc. .php?id=220\&article=50 >. Acesso em: 20 jan 2006.

GUETHES M, FLOR DP. Os principais exercícios aeróbicos. Disponível em: <http://www.efartigos.artspace.org/fitness/ artigo17.html >. Acesso em: mai 2007 
MCARDLE WD, KATCH FI, KATCH VL. Fisiologia do exercício: energia, nutrição e desempenho humano. $3^{\text {a }}$ ed. Rio de Janeiro: Guanabara Koogan, 2003.

NOVAES JV, VILHENA J. De Cinderela a Moura torta: sobre a relação mulher, beleza e feiúra. Disponível em: <www.smarcos.br/interacoes/arquivos/artigo_15>. Acesso em: 20 jan 2007.

SILVA ASR, SANTHIAGO V, GOBATTO CA. Compreendendo o overtraining no desporto: da definição ao tratamento. Disponível em: <http://www.scielo.oces.mctes.pt/scielo.php?script=sci_arttext\&pid=S1645-05232006000200011\& Ing=pt\&nrm=iso $>$. Acesso em: 20 jan 2007.

TREMBLAY A, SIMONEAU JA, BOUCHARD C. Impact of exercise intensity on body fatness and skeletal muscle metabolism. Metabolism 1994;43 (7):814-8.

WILMORE JH, COSTILL D. Fisiologia do esporte e do exercício. 1ª ed. São Paulo: Editora Manole, 2001.

\section{Endereço para correspondência:}

Fernanda Gonçalves Gomes Alvarez Rua José Vicente, 32 apto 701 - Grajaú - RJ

CEP 20540-330

Tel.: 2268-4682/2598-4402/9124-8118 e-mail: fernandaalvarez@yahoo.com

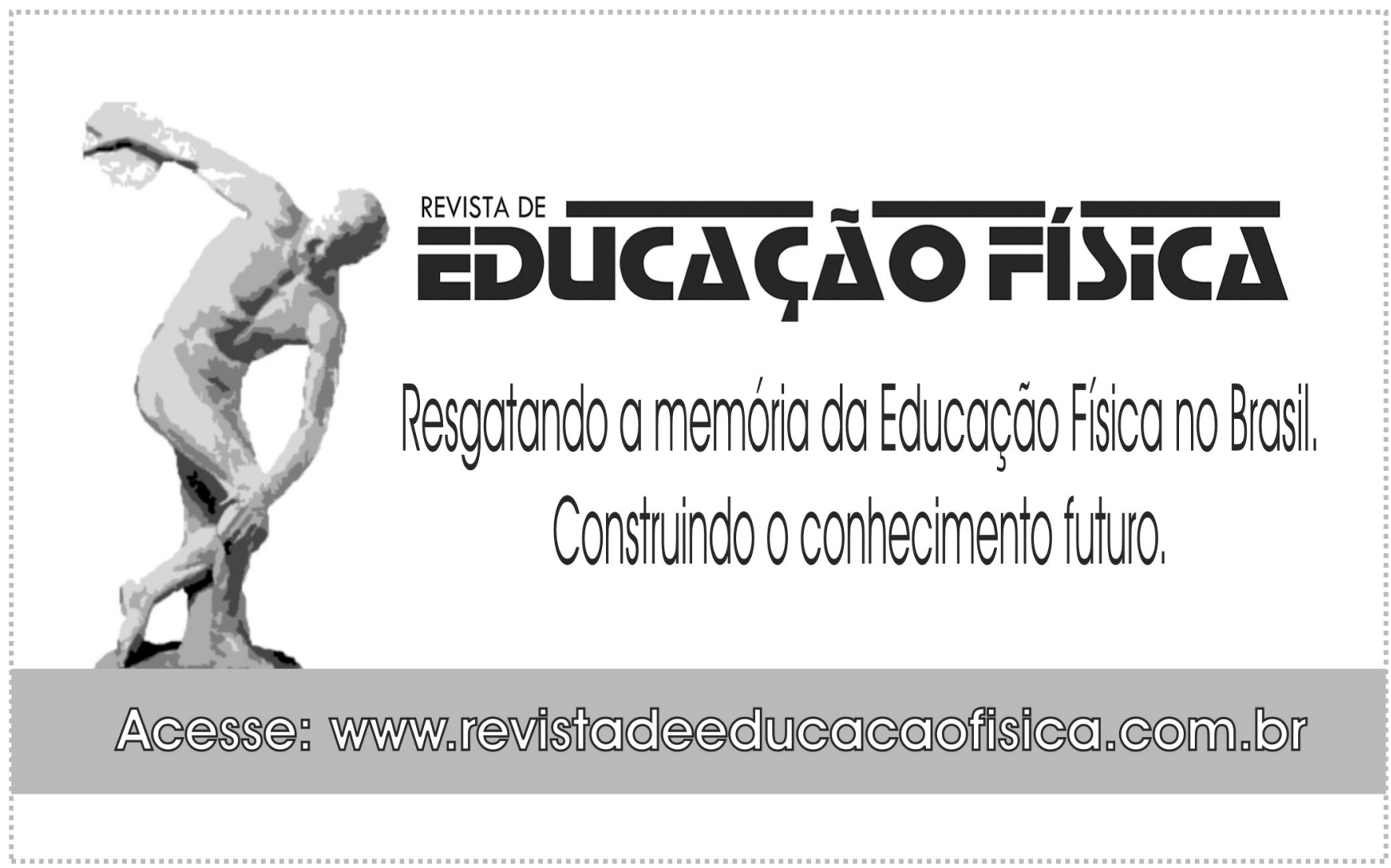

